\title{
THE SEMIGROUP OF NONEMPTY FINITE SUBSETS OF INTEGERS
}

\author{
REUBEN SPAKE \\ Department of Mathematics, \\ University of California \\ Davis, California 95616 U.S.A. \\ (Received December 16, 1985 and in revised form February 13, 1986)
}

ABSTRACT. Let $Z$ be the additive group of integers and $\delta$ the semigroup consisting of all nonempty finite subsets of $z$ with respect to the operation defined by

$$
A+B=\{a+b: a \in A, b \in B\}, A, B \in \&
$$

For $X \in 8$, define $A_{X}$ to be the basis of $\langle X-\min (X)\rangle$ and $B_{X}$ the basis of $\langle\max (X)-X\rangle$. In the greatest semilattice decomposition of $\delta$, let $a(X)$ denote the archimedean component containing $X$ and define $a_{0}(X)=\{Y \in a(X): \min (Y)=0\}$. In this paper we examine the structure of $\delta$ and determine its greatest semilattice decomposition. In particular, we show that for $X, Y \in \mathcal{S}, a(X)=a(Y)$ if and only if $A_{X}=A_{Y}$ and $B_{X}=B_{Y}$. Furthermore, if $X \in \&$ is a non-singleton, then the idempotent-free $a(X)$ is isomorphic to the direct product of the (idempotent-free) power joined subsemigroup $a_{0}(X)$ and the group $Z$.

KEY WORDS AND PHRASES. Greatest semilattice decomposition, archimedean component. 1980 AMS SUBJECT CLASSIFICATION CODE. 20M14, 10L10, 05 A99.

1. INTRODUCTION.

Let $Z$ be the group of integers and $\mathbf{g}$ the semigroup consisting of all nonempty finite subsets of $Z$ with respect to the operation defined by

$$
A+B=\{a+b: a \in A, b \in B\}, A, B \in \delta \text {. }
$$

The semigroup 8 is clearly commutative and is a subsemigroup of the power semigroup of the group of integers, (the semigroup of all nonempty subsets of $Z$ ). In this paper we will determine the greatest semilattice decomposition of 8 and describe the structure of the archimedean components in this decomposition. As we will soon see, there is a surprisingly simple necessary and sufficient condition for two elements to be in the same component.

For $\mathrm{X}=\left\{\mathrm{x}_{1}, \ldots, \mathrm{x}_{\mathrm{n}}\right\} \in \mathrm{g}$, where $\mathrm{x}_{1}<\ldots<\mathrm{x}_{n}$, define $\min (\mathrm{X})=\mathrm{x}_{1}$, 
$\max (x)=x_{n}$, and $\operatorname{gcd}(x)$ to be the greatest (non-negative) common divisor of the integers $x_{1}, \ldots, x_{n}, \quad$ (where $\left.\operatorname{gcd}(0)=0, \operatorname{gcd}(x \cup\{0\})=\operatorname{gcd}(X)\right)$. A singleton element of 8 will be identified with the integer it contains. Let $z_{+}$be the set of positive integers and define $[a, b]=\{x \in z: a \leq x \leq b\}$ if $a, b \varepsilon z$ with $a \leq b$. For $U \varepsilon \&$, let $\langle U\rangle$ denote the semigroup generated by the set $U$, and for $m \varepsilon z_{+}$define $m U, m^{*} U$, and $z_{m}$ as follows:

$$
\mathrm{mU}=\underbrace{U+\ldots+U}_{\mathrm{m}}, \mathrm{m}^{*} \mathrm{U}=\{\mathrm{mu}: \mathrm{u} \varepsilon \mathrm{U}\} \text {, and } \mathrm{Z}_{\mathrm{m}}=\mathrm{Z} /\langle-\mathrm{m}, \mathrm{m}\rangle \text {. }
$$

It will also be convenient to define $-U=\{-u: u \varepsilon U\}$.

In the greatest semilattice decomposition of $\mathbf{8}$, let $\mathbf{a}(\mathrm{A})$ denote the archimedean component containing A. As usual, define the partial order $\leq$ on the (lower) semilattice as: $a(A) \leq a(B)$ if and only if $n A=B+C$ for some $C \& 8$ and $n \in Z_{+}$(equivalently: $X+Y \varepsilon a(A)$ for some (all) $X \varepsilon Q(A)$ and $Y \varepsilon a(B)$ ). We refer the reader to Clifford and Preston [2] and Petrich [3] for more on the greatest semilattice decomposition of a commutative semigroup. Observe that since 0 is the only idempotent and indeed the identity, $a(A)$ is idempotent-free if $A$ is a non-singleton, $(a(0)$ consists of all the singletons in 8 and in fact $a(0) \cong z)$. Furthermore, if follows that the subgroups of $\delta$ are of the form $\{\{\mathrm{gx}\}: \mathrm{x} \varepsilon \mathrm{Z}\}$, where $g$ is a non-negative integer. Finally, note that 8 is clearly countable, but this of course does not imply that there are also infinitely many archimedean components. However, as will soon be shown, there are in fact infinitely many components.

2. GREATEST SEMILATTICE DECOMPOSITION.

For $X \in \delta$, define $A_{X}$ to be the basis of $\langle X-\min (X)\rangle$ and $B_{X}$ the basis of $\langle\max (X)-X\rangle$. Note that $A_{X}=B_{X}=\{0\}$ if and only if $X$ is a singleton. Also observe that $A_{X}$ is a finite set with at most $a+1$ elements, where $a$ is the least positive integer in $A_{X}$ (if $A_{X} \neq\{0\}$ ), and similarly for $B_{X}$. Since $\operatorname{gcd}(x-\min (X))=\operatorname{gcd}(X-\max (X))$, it follows that in general $\operatorname{gcd}\left(A_{X}\right)=\operatorname{gcd}\left(B_{X}\right)$.

Given sets $A$ and $B$, it is clearly not always possible to find an $X$ such that ${ }^{A} X=A$ and $B_{X}=B$. However, we do have a positive result. First we need the following lemma.

LEMMA 2.1. Let $S$ be a positive integer semigroup with respect to addition. The following are equivalent. $S$ contains $m$ such that $x \geq m$ implies $x \in S$.

(ii) $\quad \operatorname{gcd}(S)=1$.

(iii) If $\ell$ is the least element of $S$, then $S$ contains 


$$
c_{0}, \ldots, c_{\ell-1} \text { such that } c_{i} \equiv i(\bmod \ell) \text { for } i \varepsilon[0, \ell-1]
$$

PROOF. Clearly (i) implies (ii), since if $\mathrm{m}, \mathrm{m}+1 \varepsilon \mathrm{S}$, then $\mathrm{gcd}(\mathrm{S})=1$. Next suppose $\operatorname{gcd}(S)=1$ and let $B=\left\{b_{1}, \ldots, b_{n}\right\}$ be a basis with $b_{1}<\ldots<b_{n}$. If $b_{1}=1$, then evidently ( $i$ ii) follows. Thus assume $b_{1}>1$. This implies $n>1$ and hence there exist $x_{1}, \ldots, x_{n}$ such that $\sum_{i=1}^{n} x_{i} b_{i}=1$. Choose $y_{i}>0$ such that $y_{i} \equiv x_{i}\left(\bmod b_{1}\right)$ for $i \varepsilon[1, n]$. Let $c_{0}=b_{1}$ and for $i \varepsilon\left[1, b_{1}-1\right]$ define $c_{i}=i \sum_{j=1}^{n} y_{j} b_{j}$. Note that $c_{i} \varepsilon S$. Furthermore, $c_{i} \equiv i\left(\bmod b_{1}\right)$; since, $c_{0} \equiv 0$ $\left(\bmod b_{1}\right)$ and for $i \varepsilon\left[1, b_{1}-1\right]$ :

$$
c_{i}=i\left(\sum_{j=1}^{n} x_{j} b_{j}+\sum_{j=1}^{n}\left(y_{j}-x_{j}\right) b_{j}\right) \equiv i\left(\bmod b_{1}\right)
$$

Therefore (ii) implies (iii). Finally, suppose (iii) holds. Let $m=\max \left\{c_{0}, \ldots, c_{\ell-1}\right\}$ and $x \geq m$. There exists an $i \varepsilon[0, \ell-1]$ such that $x \equiv i(\bmod \ell)$. Thus $x=c_{i}+k \ell$ for some $k \varepsilon z$. However, since $x \geq c_{i}$ this implies $k \geq 0$ and hence $x \in S$. This completes the proof.

PROPOSITION 2.2. Let $A=\left\{a_{1}, \ldots, a_{n}\right\}$ and $B=\left\{b_{1}, \ldots, b_{m}\right\}$ be elements of 8 satisfying

$$
\begin{aligned}
& a_{1}=b_{1}=0, a_{1}<\ldots<a_{n}, \quad b_{1}<\ldots<b_{m}, \\
& \operatorname{gcd}(A)=\operatorname{gcd}(B), \\
& a_{i} \notin\left\langle a_{1}, \ldots, a_{i-1}\right\rangle, b_{j} \varepsilon\left\langle b_{1}, \ldots, b_{j-1}\right\rangle \text { for } i \varepsilon[2, n], j \varepsilon[2, m] .
\end{aligned}
$$

Then there exists an $r$ such that $X=A \cup(r-B)$ is an element of 8 with $A_{X}=A$ and $B_{X}=B$.

PROOF. For the case where $\operatorname{gcd}(A)=0, X=\{r\}$ is an element with $A_{X}=A$ and $B_{X}=B$, since necessarily $A=B=\{0\}$. Thus we assume $\operatorname{gcd}(A)>0$. Let $A_{1}$ and $B_{1}$ be such that $A=g * A_{1}$ and $B=g{ }^{*} B_{1}$, where $g=\operatorname{gcd}(A)$. Since $\operatorname{gcd}\left(A_{1}\right)=$ $\operatorname{gcd}\left(B_{1}\right)=1$, there exists a positive integer $q$ such that $s \in\left\langle A_{1}\right\rangle$ and $s \in\left\langle B_{1}\right\rangle$ for all $s \geq q$. Let $p=q+\max \left\{\max \left(A_{1}\right), \max \left(B_{1}\right)\right\}$. Then $p-a \varepsilon\left\langle B_{1}\right\rangle$ and $p-b \varepsilon\left\langle A_{1}\right\rangle$ for all $a \varepsilon A_{1}, b \varepsilon B_{1}$. Hence, if $r=g p$, then $r-a \varepsilon\langle B\rangle$ and $r-b \varepsilon\langle A\rangle$ for all a $\varepsilon A, b \in B$. Since $r>\max \left\{a_{n}, b_{m}\right\}$ it follows that $X=A u(r-B) \subset\langle A\rangle$ and $\max (X)-X=B u(r-A) \subset\langle B\rangle$. By the definition of $A$ and $B$, evidently $A_{X}=A$ and $B_{X}=B$. 
The next result is the key theorem which gives a necessary and sufficient condition for two elements of $\mathbf{8}$ to be in the same archimedean component.

THEOREM 2.3. For $X, Y \in 8, a(X)=a(Y)$ if and only if $A_{X}=A_{Y}$ and $B_{X}=B_{Y}$

PROOF. Suppose $X, Y \in \&$ with $A_{X}=A_{Y}$ and $B_{X}=B_{Y}$. Without loss of generality, assume $\min (X)=\min (Y)=0$ and $\max (X)=\max (Y)$. If $\operatorname{gcd}\left(A_{X}\right)=0$, then $X$ and $Y$ are singletons and thus $a(X)=a(Y)$. So assume $g c d\left(A_{X}\right)>0$. Let $U$ and $V$ be such that $X=g^{*} U$ and $Y=g * V$, where $g=\operatorname{gcd}\left(A_{X}\right)$. Note that $\operatorname{gcd}\left(A_{U}\right)=1$. Let $a$ and $b$ be the least positive integers in $A_{U}$ and $B_{U}$, respectively. Define $A_{i}=\left\{x \in\left\langle A_{U}\right\rangle: x \equiv i(\bmod a)\right\}$ and $B_{j}=\left\{x \varepsilon\left\langle B_{U}\right\rangle: x \equiv j\right.$ $(\bmod b)\}$ for $i \varepsilon[0, a-1], j \varepsilon[0, b-1]$. Also, define $c_{i}=\min \left(A_{i}\right), d_{i}=$ $\min \left(B_{i}\right), c=\max \left\{c_{i}: i \varepsilon[0, a-1]\right\}$, and $d=\max \left\{d_{i}: i \varepsilon[0, b-1]\right\}$. Choose m, $r \varepsilon Z_{+}$such that

$$
\begin{aligned}
& \max \{c, d\}+\max (U) \leq(m+1) \min \{a, b\}, \\
& c_{i} \varepsilon \mathrm{rU} \text { for all i } \varepsilon[0, a-1],
\end{aligned}
$$$$
d_{i} \varepsilon r(\max (U)-U) \text { for all i } \varepsilon[0, b-1] \text {. }
$$

Finally, let $n=m+r$.

By the definition of $n$, evidently

$$
\begin{aligned}
& \underset{i=0}{u-1}\left\{x \in A_{i}: x<c-a\right\} \cup[c-a+1, m a] \\
& \quad \therefore \quad \underset{j=0}{u} u_{i=0}^{a-1}\left\{c_{i}+j a\right\} \subset n U
\end{aligned}
$$

and similarly

$$
\bigcup_{i=0}^{b-1}\left\{x \quad \varepsilon \quad B_{i}: x<d-b\right\} \quad u[d-b+1, m b] \subset n(\max (U)-U) .
$$

Also, observe that $c-a \notin s U$ and $d-b \notin s(\max (U)-U)$ for all $s \varepsilon Z_{+}$(by definition). Since $c+\max (U) \leq(m+1) a$ and $d+\max (U) \leq(m+1) b$, it follows that for all $p \geq 0$

$$
\begin{aligned}
& \underbrace{p}_{i=0}[c-a+1+i \max (U), \operatorname{ma}+i \max (U)] \\
& \quad=[c-a+1, \operatorname{ma}+p \max (U)] c(n+p) U
\end{aligned}
$$

and similarly 


$$
[d-b+1, m b+p \max (U)] c(n+p)(\max (U)-U)
$$

Thus, for all $\mathrm{q} \geq \mathrm{n}$

$$
\begin{aligned}
& {[c-a+1, \operatorname{ma}+(q-n) \max (U)] u} \\
& {[n \max (U)-m b, q \max (U)+b-d-1] \subseteq q U .}
\end{aligned}
$$

In particular,

$$
\begin{aligned}
& {[c-a+1, \operatorname{ma}+n \max (U)] U} \\
& {[n \max (U)-m b, 2 n \max (U)+b-d-1]} \\
& =[c-a+1,2 n \max (U)+b-d-1] \subseteq 2 n U .
\end{aligned}
$$

It is clear that if $u \in q U$ with $u<c-a$ and $q \geq n$, then

$$
u \varepsilon \bigcup_{i=0}^{a-1}\left\{x \in A_{i}: x<c-a\right\}
$$

Likewise if $u \in q U$ with $u>q \max (U)+b-d$ and $q \geq n$, then

$$
u \varepsilon \underset{i=0}{u-1}\left\{q \max (U)-x: x \in B_{i}, x<d-b\right\} .
$$

Hence

$$
\begin{aligned}
2 n U= & \underset{i=0}{u-1}\left\{x \quad \varepsilon \quad A_{i}: x<c-a\right\} \quad u[c-a+1,2 n \max (U)+b-d-1] \\
& \underset{i=0}{u-1}\left\{2 n \max (U)-x: x \in B_{i}, x<d-b\right\}=2 n V .
\end{aligned}
$$

Therefore, $2 \mathrm{nX}=2 \mathrm{nY}$ and $a(\mathrm{X})=a(\mathrm{Y})$.

Conversely, suppose $a(X)=a(Y)$. Then there exist $S, T \varepsilon \&$ and $s, t \in Z_{+}$such that

$$
\begin{aligned}
& \mathrm{S}(\mathrm{X}-\min (\mathrm{X}))=\mathrm{Y}-\min (\mathrm{Y})+\mathrm{S} \text { and } \\
& \mathrm{t}(\mathrm{Y}-\min (\mathrm{Y}))=\mathrm{X}-\min (\mathrm{X})+\mathrm{T} .
\end{aligned}
$$

Since necessarily $\min (S)=\min (T)=0$, it follows that

$$
A_{Y} \subseteq Y-\min (Y)+S \subseteq\left\langle A_{X}\right\rangle
$$

and similarly $A_{X} \subseteq\left\langle A_{Y}\right\rangle$. Consequently, $\left\langle A_{X}\right\rangle=\left\langle A_{Y}\right\rangle$ and hence by the definition of $A_{X}$ and $A_{Y}$ we have $A_{X}=A_{Y}$. Similarly it is easy to show $B_{X}=B_{Y}$ and this completes the proof.

Perhaps a brief example will help illustrate the simplicity of the condition given in Theorem 2.3. Let $W=\{-10,-8,22,55,57\}, X=\{3,5,29,68,69\}$, and $Y=\{4,6,69,85,86\}$. Then 


$$
\begin{aligned}
& W-\min (W)=\{0,2,32,65,67\}, \max (W)-W=\{0,2,35,65,67\}, \\
& X-\min (X)=\{0,2,26,65,66\}, \max (X)-X=\{0,1,40,64,66\}, \\
& Y-\min (Y)=\{0,2,65,81,82\}, \max (Y)-Y=\{0,1,17,80,82\} .
\end{aligned}
$$

Hence, $A_{W}=A_{X}=A_{Y}=\{0,2,65\}, B_{W}=\{0,2,35\}$, and $B_{X}=B_{Y}=\{0,1\}$. Therefore $a(X)=a(Y)$ and $a(W) \neq a(X)$. Actually, $a(X) \leq a(W)$ by our next theorem.

Using Theorem 2.3 we can determine when two archimedean components are related with respect to the order on the semilattice.

THEOREM 2.4. The following are equivalent.

$$
a(X) \leq a(Y) \text {. }
$$

$$
\begin{aligned}
& A_{Y} \subseteq\left\langle A_{X}\right\rangle \text { and } B_{Y} \subseteq\left\langle B_{X}\right\rangle . \\
& { }_{X+Y}=A_{X} \text { and } B_{X+Y}=B_{X} .
\end{aligned}
$$

PROOF. Suppose $a(X) \leq a(Y)$. There exist $U \in \&$ and $n \varepsilon Z_{+}$such that

$$
\mathrm{n}(\mathrm{X}-\min (\mathrm{X}))=\mathrm{Y}-\min (\mathrm{Y})+\mathrm{U} \text {. }
$$

Since $\min (U)=0$,

$$
A_{Y} \subseteq Y-\min (Y)+U \subseteq\left\langle A_{X}\right\rangle
$$

Similarly $B_{Y} \subseteq\left\langle B_{X}\right\rangle$. Suppose next that assertion (ii) holds. Then

$$
Y-\min (Y) \subseteq\left\langle A_{Y}\right\rangle\left\langle A_{X}\right\rangle
$$

and thus

$$
X+Y-\min (X+Y)=A_{X} \cup X_{1}
$$

where $X_{1} \subseteq\left\langle A_{X}\right\rangle$. Hence $A_{X+Y}=A_{X}$. Likewise $B_{X+Y}=B_{X}$. Finally, if (iii) holds, then by Theorem 2.3 X+Y $\varepsilon a(X)$; that is, $a(X) \leq a(Y)$ and the proof is complete.

Observe that clearly $A_{Y} \subseteq A_{X}$ and $B_{Y} \subseteq B_{X}$ is a sufficient condition for $a(X) \leq a(Y)$. However, it is not a necessary condition (see Spake [4]). Since $A_{Y}$ and $B_{Y}$ are finite sets, it is relatively easy to determine when $a(X) \leq a(Y)$ via Theorem 2.4 (ii). Also, as the trivial case of Theorem 2.4, we have $a(0,1) \leq a(x) \leq$ $a(0)$ for all $x \in \delta$ and hence $a(0,1)$ is an ideal of $\boldsymbol{s}$.

Define $a_{0}(X)=\{Y \varepsilon a(X): \min (Y)=0\}$ and note that $a_{0}(X)$ is a subsemigroup of $a(x)$. Moreover, since elements of $a(x)$ can be uniquely expressed in the form $U+a$, where $U \in a_{0}(X)$ and $a \in z$, evidently $a(X) \cong a_{0}(X) \oplus z$. Recalling the proof of Theorem 2.3, apparently if $x$ is a non-singleton, then $a_{0}(x)$ is power joined. We therefore immediately have 
THEOREM 2.5. The idempotent-free archimedean component $a(X)$, where $X$ is a non-singleton, is isomorphic to the direct product of the idempotent-free power joined subsemigroup $a_{0}(X)$ and the group $Z$.

We complete this section with a brief summary of the greatest semilattice decomposition of $\mathbf{s}$. Let

$$
\begin{aligned}
W=\left\{\left(\left(a_{1}, \ldots, a_{n} ; b_{1}, \ldots, b_{m}\right)\right):\right. & a_{i}, b_{j} \varepsilon z, 0=a_{1}<\ldots<a_{n}, 0=b_{1}<\ldots<b_{m}, \\
& \operatorname{gcd}\left(a_{1}, \ldots, a_{n}\right)=\operatorname{gcd}\left(b_{1}, \ldots, b_{m}\right), \\
& a_{i} \varepsilon\left\langle a_{1}, \ldots, a_{i-1}\right\rangle \text { and } b_{j} \varepsilon\left\langle b_{1}, \ldots, b_{j-1}\right\rangle, \\
& \text { for i } i[2, n], j \varepsilon[2, m]\} .
\end{aligned}
$$

Define a partial order $\leq$ on $W$ as follows:

$$
\begin{aligned}
& \left(\left(a_{1}, \ldots, a_{n} ; b_{1}, \ldots, b_{m}\right)\right) \leq\left(\left(c_{1}, \ldots, c_{p} ; d_{1}, \ldots, d_{q}\right)\right) \text { if and only if } \\
& \left\{c_{1}, \ldots, c_{p}\right\} \subseteq\left\langle a_{1}, \ldots, a_{n}\right\rangle \text { and }\left\{d_{1}, \ldots, d_{q}\right\} \subseteq\left\langle b_{1}, \ldots, b_{m}\right\rangle \text {. }
\end{aligned}
$$

Also, define the map $\Phi: s+w$ by $\Phi(X)=\left(\left(a_{1}, \ldots, a_{n} ; b_{1}, \ldots, b_{m}\right)\right)$ where $\left\{a_{1}, \ldots, a_{n}\right\}=A_{X}$ and $\left\{b_{1}, \ldots, b_{m}\right\}=B_{X}$. Using our preceding results we have the following theorem.

THEOREM 2.6. The map $\Phi$ is the greatest semilattice homomorphism of 8 , with $W$ being the greatest semilattice homomorphic image. Moreover, if $\Phi(X)=\left(\left(a_{1}, \ldots, a_{n} ; b_{1}, \ldots, b_{m}\right)\right)$ and $\Phi(Y)=\left(\left(c_{1}, \ldots, c_{p} ; d_{1}, \ldots, d_{q}\right)\right)$, then $\Phi(X) \leq \Phi(Y)$ if and only if $\left\{c_{1}, \ldots, c_{p}\right\} \subseteq\left\langle a_{1}, \ldots, a_{n}\right\rangle$ and $\left\{d_{1}, \ldots, d_{q}\right\}$ $\left\langle b_{1}, \ldots, b_{m}\right\rangle$.

We further define two congruences $\delta$ and $\zeta$ on 8 as follows:

$$
\begin{aligned}
& X \delta Y \text { if and only if } X=Y+Z \text { for some } Z \varepsilon Z \text {, } \\
& X \zeta Y \text { if and only if } \Phi(X)=\Phi(Y) \text { and } \min (X)=\min (Y) \text {. }
\end{aligned}
$$

Observe that $\mathbf{g} / \delta$ is isomorphic to the subsemigroup $\boldsymbol{H}$ of $\mathbf{8}$ consisting of $X$ satisfying $\min (X)=0$ and $\boldsymbol{A}$ is the semilattice $W$ of $a_{0}(A)$ 's. Also, $\delta / \zeta$ is isomorphic to the direct product $K$ of $W$ and $Z$. Next, recall the definition of spined product: if $g_{i}: S_{i} \rightarrow T$ is a homomorphism of $S_{i}$ onto $T(i=1,2)$, then the spined product of $S_{1}$ and $S_{2}$ with respect to $g_{1}$ and $g_{2}$ is $\left\{(x, y): x \in S_{1}\right.$, $\left.y \in S_{2}, g_{1}(x)=g_{2}(y)\right\}$ in which $\left(x_{1}, y_{1}\right)+\left(x_{2}, y_{2}\right)=\left(x_{1}+x_{2}, y_{1}+y_{2}\right)$.

Using our results we have 
THEOREM 2.7. The semigroup $\&$ is isomorphic to the spined product of $\&$ and $x$ with respect to $\boldsymbol{H} \rightarrow W$ and $x \rightarrow W$.

3. STRUCTURE OF THE COMPONENTS.

The structure of $a(0)$ is clear, since $a(0) \cong z$. In this section we investigate the structure of $a(x)$ when $x$ is a non-singleton. We begin with a general result from Theorem 2.3 .

PROPOSITION 3.1. For $X, Y \in 8, Y \in a(X)$ if and only if

$$
\begin{aligned}
& Y-\min (Y)=A_{X} \cup X_{1}, \text { where } X_{1} \subseteq\left\langle A_{X}\right\rangle ; \text { and } \\
& \max (Y)-Y=B_{X} \cup X_{2}, \text { where } X_{2} \subseteq\left\langle B_{X}\right\rangle .
\end{aligned}
$$

For $\mathrm{x}=\left\{\mathrm{x}_{1}, \ldots, \mathrm{x}_{\mathrm{n}}\right\} \in \mathrm{s}$, where $\mathrm{n}>1$ and $\mathrm{x}_{1}<\ldots<\mathrm{x}_{\mathrm{n}}$, define id $(\mathrm{x})=$ $x_{2}-x_{1}$ and $f d(x)=x_{n}-x_{n-1}$. Notice that $i d(x)$ and $f d(x)$ are the least positive integers in ${ }^{A} X$ and ${ }^{B} X$, respectively. Recalling the proof of Theorem 2.3 , we evidently have

THEOREM 3.2. Let $X$ be a non-singleton and $U$ be such that $X-m i n(X)=g^{*} U$, where $g=\operatorname{gcd}\left(A_{X}\right)$. Define $A_{i}=\left\{x \varepsilon\left\langle A_{U}\right\rangle: x \equiv i(\bmod a)\right\}$ and $B_{j}=\left\{x \varepsilon\left\langle B_{U}\right\rangle:\right.$ $x \equiv j(\bmod b)\}$ for $i \varepsilon[0, a-1], j \varepsilon[0, b-1]$, where $a=i d(U)$ and $b=f d(U)$. Let $c=\max \left\{\min \left(A_{i}\right): i \varepsilon[0, a-1]\right\}$ and $d=\max \left\{\min \left(B_{i}\right): i \varepsilon[0, b-1]\right\}$. Then $Y \varepsilon a(X)$ if and only if there exist $V \in \&$ and $n_{0} \varepsilon Z_{+}$such that $Y-m i n(Y)=g^{* V}$ and for all $n \geq n_{0}$

$$
\begin{aligned}
& n V=\underset{i=0}{u-1}\left\{x \in E A_{i}: x<c-a\right\} \cup[c-a+1, n \max (V)+b-d-1] \\
& u_{i=0}^{b-1}\left\{n \max (V)-x: x \in B_{i}, x<d-b\right\}
\end{aligned}
$$

Next we reproduce several definitions and facts from Tamura [5] that we will need in the following development. We direct the reader to [5] for a more complete discussion of the notions which follow. Let $T$ be an additively denoted commutative idempotent-free archimedean semigroup. Define a congruence $\rho_{b}$ on $T$, for fixed b, as

$$
x \rho_{b} y \text { if and only if } n b+x=m b+y \text { for some } n, m \varepsilon z_{+} \cdot
$$

Then $T / \rho_{b}=G_{b}$ is a group called the structure group of $T$ determined by the standard element b. Also, define a compatible partial order < on $T$ as follows: 
$x<y$ if and only if $x=n b+y$ for some $n \varepsilon Z_{+}$.

Then $T=\underset{\lambda \varepsilon G_{b}}{U} T_{\lambda}$, equivalently $T / \rho_{b}=\left\{T_{\lambda}\right\}, \quad \lambda \varepsilon G_{b}$, where each $T_{\lambda}$ is a lower semilattice with respect to $\underset{b}{<}$ In fact, for each $\lambda \varepsilon G_{b}, T_{\lambda}$ forms a discrete tree without smallest element with respect to $\underset{b}{<}$, (a discrete tree, with respect to $<$, is a lower semilattice such that for any $c<d$ the $\operatorname{set}\{x: c<x<d\}$ is a $b \quad b \quad b \quad b$ finite chain). Finally, we define a relation $n$ on $T$ as follows:

$$
x \cap y \text { if and only if } n b+x=n b+y \text { for some } n \varepsilon z_{+} \text {. }
$$

The relation $\eta$ is the smallest cancellative congruence on $T$.

We continue our development with the following theorem.

THEOREM 3.3. Let $A \in \mathbf{8}$ be a non-singleton with $\min (\mathrm{A})=0$ and $g=\operatorname{gcd}(\mathrm{A})$. The structure group of $a_{0}(A)$ determined by the standard element $A$ is $Z_{m}$, where $m=\max (A) / g$. Moreover, $a_{0}(A)=\bigcup_{i=0}^{m-1} a_{i}$ where $a_{i}=\left\{x \in a_{0}(A): \max (x) / g \equiv i\right.$ $(\bmod \mathrm{m})\} \quad$ is a discrete tree without smallest element with respect to

Furthermore, the structure group of $a(A)$ determined by the standard element $A$ is $\mathrm{Z} \oplus \mathrm{Z}_{\mathrm{m}}$.

PROOF. Let $U, V \in a_{0}(A)$ and $C, U_{1}, V_{1}$ be such that $A=g^{*} C, U=g^{*} U_{1}$, and $V=g^{*} V_{1}$, where $g=\operatorname{gcd}(A)$. For $i \varepsilon[0, a-1], j \varepsilon[0, b-1]$, where $a=i d(C)$ and $b=f d(C)$, define $A_{i}=\left\{x \varepsilon\left\langle A_{C}\right\rangle: x \equiv i(\bmod a)\right\}$ and $B_{j}=\left\{x \in\left\langle B_{C}\right\rangle: x \equiv j(\bmod b)\right\}$. Also, let $c=\max \left\{\min \left(A_{i}\right): i \varepsilon[0, a-1]\right\}$ and $d=\max \left\{\min \left(B_{i}\right): i \varepsilon[0, b-1]\right\}$.

Suppose $\max \left(U_{1}\right) \equiv \max \left(V_{1}\right)(\bmod m)$, where $m=\max (C)$. Without loss of generality, assume $\max \left(U_{1}\right)=\max \left(V_{1}\right)+k m$ with $k \geq 0$. There exists $p \geq c+d+\max \left(U_{1}\right)$ such that

$$
\begin{gathered}
p C=\bigcup_{i=0}^{a-1}\left\{x \in A_{i}: x<c-a\right\} \cup[c-a+1, p m+b-d-1] \\
\qquad \underset{i=0}{b-1}\left\{p m-x: x \in B_{i}, x<c-b\right\} .
\end{gathered}
$$


Since $U_{1} \varepsilon a_{0}(c)$, it follows that

$$
\begin{aligned}
& U_{1} \subseteq \bigcup_{i=0}^{a-1}\left\{x \quad \varepsilon \quad A_{i}: x<c-a\right\} \cup\left[c-a+1, \max \left(U_{1}\right)+b-d-1\right] \\
& \bigcup_{i=0}^{b-1}\left\{\max \left(U_{1}\right)-x: x \in B_{i}, x<d-b\right\}
\end{aligned}
$$

and similarly for $V_{1}$. Hence

$$
\begin{aligned}
& U_{1}+p C=\bigcup_{i=0}^{a-1}\left\{x \in A_{i}: x<c-a\right\} U\left[c-a+1, p m+\max \left(U_{1}\right)+b-d-1\right] \\
& u \underset{i=0}{u-1}\left\{p m+\max \left(U_{1}\right)-x: x \in B_{i}, x<d-b\right\} \\
& a-1 \\
& =\bigcup_{i=0}\left\{x \in A_{i}: x<c-a\right\} \cup\left[c-a+1,(p+k) m+\max \left(V_{1}\right)+b-d-1\right] \\
& u \underset{i=0}{u-1}\left\{(p+k) m+\max \left(v_{1}\right)-x: x \in B_{i}, x<d-b\right\} \\
& =v_{1}+(p+k) C \text {. }
\end{aligned}
$$

Consequently, $U+p A=V+(p+k) A$.

Conversely, if $U+r A=V+s A$ for some $r, s \varepsilon Z_{+}$, then

$$
\max (U)+r g m=\max (V)+\mathrm{sgm} .
$$

Since $g / \max (U)$ and $g / \max (V)$, evidently $\max (U) / g \equiv \max (V) / g(\bmod m)$. By Proposition 3.1, if $t \geq \max \left\{\max \left(A_{C}\right)+d-b+1, \max \left(B_{C}\right)+c-a+1\right\}$ and $t \varepsilon Z_{+}$, then $X=A_{C} U\left(t-B_{C}\right) \in a_{0}(C)$ with $\max (x)=t$. It follows that for each $i \varepsilon Z_{m}$, there exists $X \in Q_{0}(A)$ with $\max (X) / g \equiv i(\bmod m)$. Therefore, the structure group of $a_{0}(\mathrm{~A})$ determined by the standard element $A$ is $Z_{m}$. Using the above, it is clear that for $X, Y \varepsilon a(A)$,

$$
\begin{aligned}
& X+r A=Y+s A \text { for some, } r, s \in Z_{+} \text {if and only if } \min (X)=\min (Y) \\
& \text { and }(\max (X)-\min (X)) / g \equiv(\max (Y)-\min (Y)) / g(\bmod m) \text {. }
\end{aligned}
$$

This completes the proof.

We conclude this paper with two related propositions. 
PROPOSITION 3.4. Let $X$ be a non-singleton. The homomorphism $h: a_{0}(x) \rightarrow z_{+}$ defined by $h(U)=\max (U)$ is the greatest cancellative homomorphism. That is, the relation $n$ on $a_{0}(x)$ defined by

$$
\mathrm{U} \cap \mathrm{V} \text { if } \text { and only if } \max (\mathrm{U})=\max (\mathrm{V})
$$

is the smallest cancellative congruence. Furthermore, the relation 0 on $a(X)$ defined by

$$
U \circ V \text { if } \frac{\text { and }}{\text { only }} \frac{\text { if }}{\min (U)=\min (\mathrm{V}) \text { and }}
$$

is the smallest cancellative congruence. The semigroups $a_{0}(x) / n$ and $a(x) / 0$ are $\mathfrak{n}$-semigroups.

PROPOSITION 3.5. Let $X$ be a non-singleton and $U$ be such that $X-m i n(X)=$ $g^{*} U$, where $g=\operatorname{gcd}\left(A_{X}\right)$. For $i \varepsilon[0, a-1], j \varepsilon[0, b-1]$, where $a=i d(U)$ and $b=f d(U)$, define $c_{i}$ and $d_{j}$ to be the least integers in $\left\langle A_{U}\right\rangle$ and $\left\langle B_{U}\right\rangle$, respectively, such that $c_{i} \equiv i(\bmod a)$ and $d_{j} \equiv j(\bmod b)$. Let $c=\max \left\{c_{i}: i \varepsilon[0, a-1]\right\}, d=\max \left\{d_{i}: i \varepsilon[0, b-1]\right\}, m=\max \left\{\max \left(A_{U}\right), \max \left(B_{U}\right)\right\}$, and $p=\max \left\{\max \left(A_{U}\right)+d-b+1, \max \left(B_{U}\right)+c-a+1\right\}$. Then the greatest cancellative homomorphic image of $a_{0}(x)$ is isomorphic to the following positive integer semigroup:

$$
\begin{aligned}
& C=\left\{r \in[m, p-2]: \text { for all } x \in A_{U}, y \in B_{U}, \text { if } r-x \equiv j(\bmod b)\right. \text {, } \\
& \text { for some } j \in[0, b-1] \text {, then } r \geq x+d j \text { and } \\
& \text { if } r-y \equiv i(\bmod a) \text {, for some } i \in[0, a-1] \text {, } \\
& \text { then } \left.r \geq y+c_{i}\right\} \\
& u\{r \in z: r \geq p\}
\end{aligned}
$$

(where if $[\mathrm{m}, \mathrm{p}-2]$ is not defined then $C=\{r \in Z: r \geq p\})$.

PROOF. First, observe that $a_{0}(X)$ and $a_{0}(U)$ have isomorphic greatest cancellative homomorphic images, since $a_{0}(X) \cong a_{0}(U)$. Let $V \in a_{0}(U)$ and $t=\max (V)$. Since $A_{U} \subseteq V$ and $B_{U} \subseteq t-V$, it follows that $t \geq \max \left\{\max \left(A_{U}\right)\right.$, $\left.\max \left(B_{U}\right)\right\}$. Moreover, using Proposition 3.1, $t-x \varepsilon\left\langle B_{U}\right\rangle$ and $t-y \varepsilon\left\langle A_{U}\right\rangle$ for all $x \in A_{U}, y \varepsilon B_{U}$. Thus, by the definition of $c_{i}$ and $d_{j}, t \varepsilon C$. Furthermore, if $r \varepsilon \mathcal{C}$ then evidently $A_{U} \cup\left(r-B_{U}\right) \in a_{0}(U)$. Consequently, the proof is complete by Proposition 3.4 . 


\section{REFERENCES}

1. TAMURA, T. and SHAFER, J. Power Semigroups, Math. Japon. 12(1967) 25-32.

2. CLIFFORD, A. H. and PRESTON, G. B. The Algebraic Theory of Semigroups, Amer. Math. Soc., 1961.

3. PETRICH, M. Introduction to Semigroups, Merrill, 1973.

4. SPAKE, R. Idempotent-free Archimedean Components of the Power Semigroup of the Group of Integers 1, to appear in Math. Japon. 31(May 1986).

5. TAMURA, T. Construction of Trees and Commutative Archimedean Semigroups, Math. Nachr. 36(1968) 255-287. 


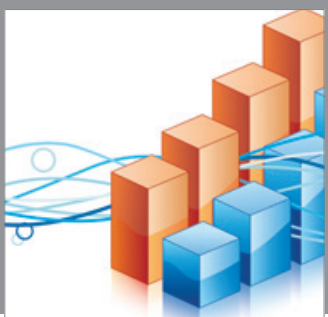

Advances in

Operations Research

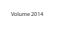

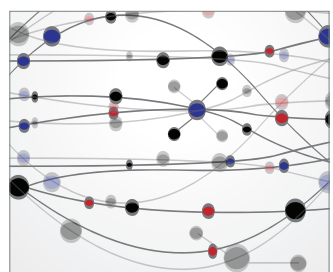

\section{The Scientific} World Journal
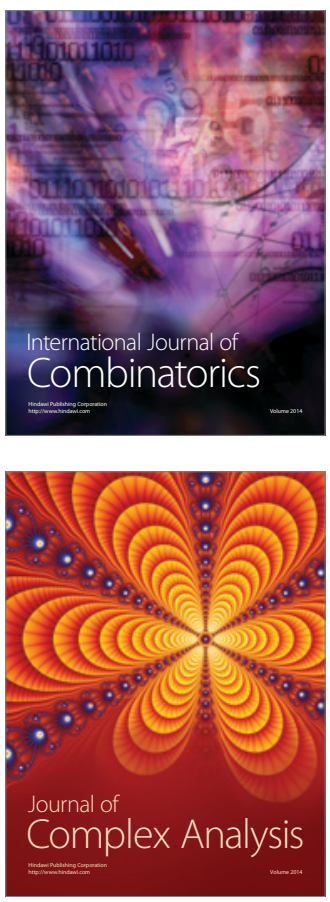

International Journal of

Mathematics and

Mathematical

Sciences
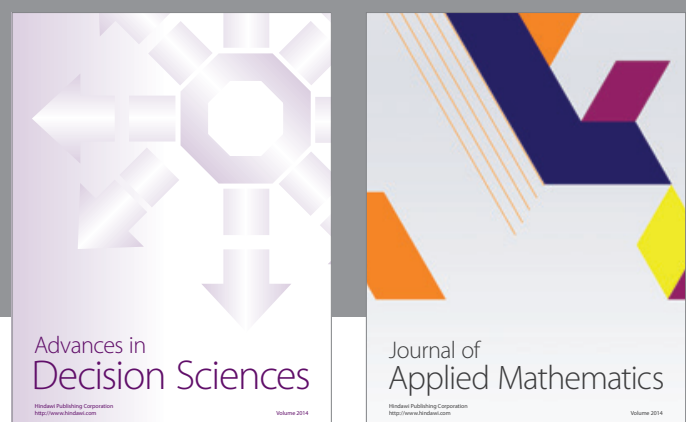

Journal of

Applied Mathematics
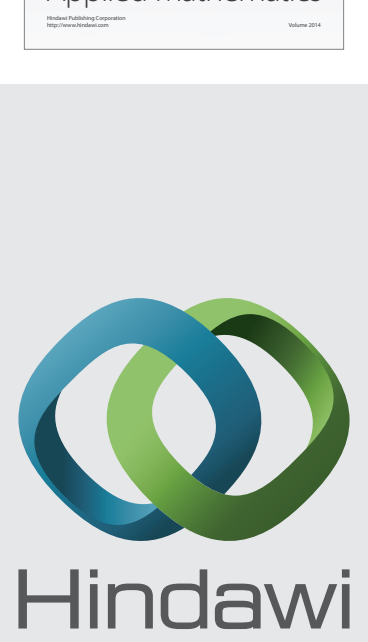

Submit your manuscripts at http://www.hindawi.com
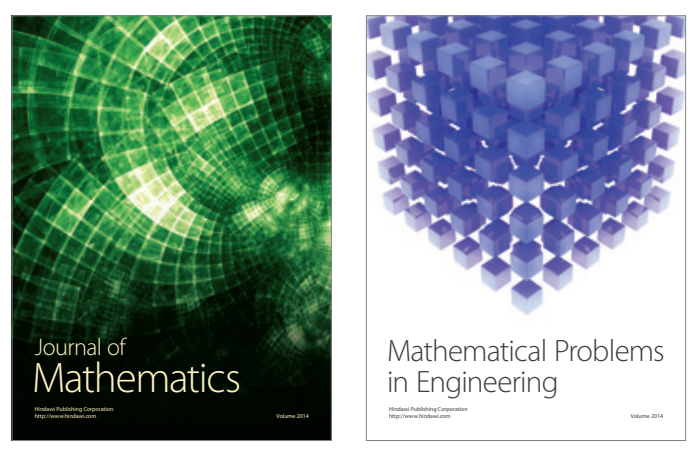

Mathematical Problems in Engineering
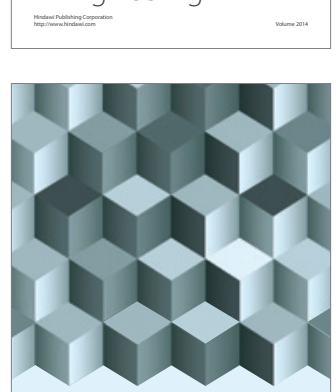

Journal of

Function Spaces
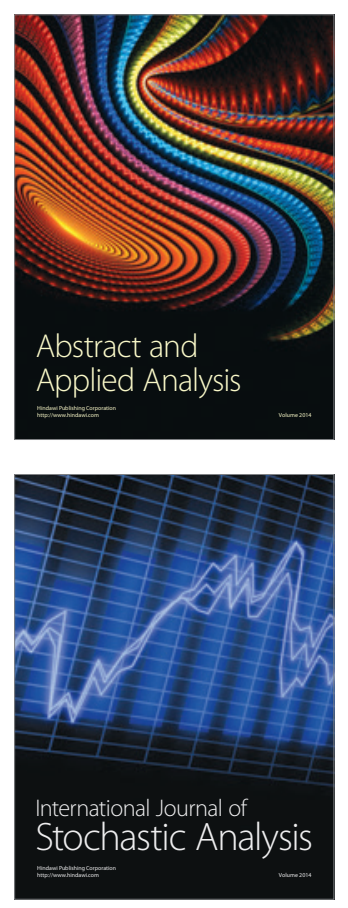

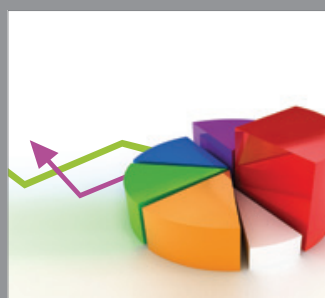

ournal of

Probability and Statistics

Promensencen
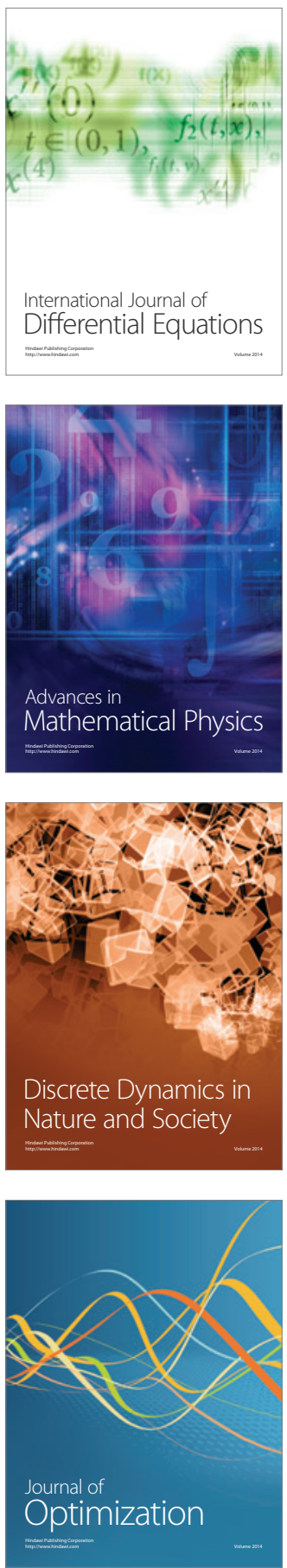